\title{
Alternative TFAP2A isoforms have distinct activities in breast cancer
}

\author{
Chiara Berlato, KaYi V Chan, Anna M Price, Monica Canosa, Angelo G Scibetta and Helen C Hurst ${ }^{*}$
}

\begin{abstract}
Introduction: AP- $2 \alpha$ is a transcription factor implicated in the regulation of differentiation and proliferation in certain tissues, including the mammary gland. In breast tumours, continued expression of AP-2 $\alpha$ has been correlated with a better prognosis, but this is hard to reconcile with a reported role in the upregulation of the ERBB2 oncogene. The existence of TFAP2A isoforms, deriving from alternative first exons and differing in their $\mathrm{N}$ terminal sequence, has been described in some mammals, but their relative abundance and activity has not been investigated in the human breast.

Methods: Expression levels of four TFAP2A isoforms were assayed at the level of RNA and protein (via the generation of isoform-specific antibodies) in a panel of breast tumour cell lines and in tissue from normal breast and primary tumour samples. Expression constructs for each isoform were used in reporter assays with synthetic and natural promoters (cyclin D3 and ERBB2) to compare the activation and repression activity of the isoforms.

Results: We demonstrate that the two isoforms AP- $2 \alpha 1 \mathrm{~b}$ and AP- $2 \alpha 1 \mathrm{c}$, in addition to the originally cloned, AP$2 \alpha 1 \mathrm{a}$, are conserved throughout evolution in vertebrates. Moreover, we show that isoform 1c in particular is expressed at levels at least on a par with the 1a isoform in breast epithelial lines and tissues and may be more highly expressed in tamoxifen resistant tumours. The isoforms share a similar transactivation mechanism involving the recruitment of the adaptors CITED2 or 4 and the transactivators p300 or CBP. However, isoform 1b and 1c are stronger transactivators of the ERBB2 promoter than isoform 1a. In contrast, AP-2 $\alpha$ 1a is the only isoform able to act as a repressor, an activity that requires an intact sumoylation motif present within the $\mathrm{N}$-terminus of the protein, and which the other two isoforms lack.

Conclusions: Our findings suggest that TFAP2A isoforms may be differentially regulated during breast tumourigenesis and this, coupled with differences in their transcriptional activity, may impact on tumour responses to tamoxifen therapy. These data also have implications for the interpretation of tumour studies that seek to correlate outcomes with TFAP2A expression level.
\end{abstract}

\section{Introduction}

AP- $2 \alpha$ belongs to the AP-2 family of transcription factors with four other members, AP- $2 \beta, \gamma, \delta$ and $\varepsilon$ [1], which have all been implicated in the regulation of proliferation and differentiation in specific tissues. In particular, AP- $2 \alpha$ is expressed in the developing and adult mammary gland $[2,3]$. In breast cancer, lower AP- $2 \alpha$ expression levels are found in invasive cancer compared to ductal carcinoma in situ (DCIS) and normal breast [4], while high levels of AP- $2 \alpha$ correlate with a more favourable outcome [2,5]. Among the known target

\footnotetext{
* Correspondence: h.c.hurst@gmul.ac.uk

Centre for Tumour Biology, Bart's Cancer Institute, Queen Mary University of London, Charterhouse Square, London EC1M 6BQ, UK
}

genes, many play a key role in breast biology and tumorigenesis. AP- $2 \alpha$ is a central player in the positive regulation of $E R B B 2$ expression [6], supported by studies demonstrating a correlation between AP- $2 \alpha$ levels and expression of the receptor in tumour samples [5,7], but in conflict with other observations [4]. How the role of AP- $2 \alpha$ as a tumour suppressor reconciles with its activity in inducing $E R B B 2$ is still unclear.

AP-2 proteins interact as homo- and hetero-dimers which bind to specific $\mathrm{GC}$-rich sequences to regulate transactivation or repression [8]. The best characterised mechanism of transactivation involves the recruitment of the transcriptional activators CBP and p300 through interaction with the small adaptor proteins CITED2 [9]
C Biomed Central

(c) 2011 Berlato et al.; licensee BioMed Central Ltd. This is an open access article distributed under the terms of the Creative Commons Attribution License (http://creativecommons.org/licenses/by/2.0), which permits unrestricted use, distribution, and reproduction in any medium, provided the original work is properly cited. 
or CITED4 [10]. The importance of these interactions in vivo is underlined by the observation that CITED2 and AP- $2 \alpha$ knockout mice have overlapping phenotypes [11]. AP-2 $\alpha$ is known to repress expression of a number of genes, including $\mathrm{C} / \mathrm{EBP} \alpha$ [12], Bcl-2 [13], EGFR [14], but the mechanism of repression is unknown. However, the related AP- $2 \gamma$ is known to interact with UBC9 and to be sumoylated, resulting in downregulation of its transcriptional activity [15].

The TFAP $2 A$ gene consists of seven exons with the last six exons encoding the majority of the protein, including the activation, DNA binding and dimerisation domains [1]. The existence of different TFAP2A isoforms deriving from alternative first exons has been described in murine embryo and HeLa cells [16], and in ovine and human placenta [17]. Some variation in spatio-temporal expression between the isoforms during murine embryonic development was identified using in situ hybridisation [16]; however, the function of these splice variants, which differ solely in the extreme aminoterminal sequence of AP- $2 \alpha$, has not been investigated further. Generally, transcripts deriving from alternative first exons are frequently observed in mammalian genomes, with an estimated $>20 \%$ of genes having active alternative promoters [18]. This contributes to the complexity of the function of a gene by providing additional levels of regulation of expression and, if the translation start site exists within the first exon as in the case of AP- $2 \alpha$, by encoding distinct proteins which potentially have quite different biological functions.

Our aim was to characterise the role of human AP- $2 \alpha$ isoforms in normal breast epithelial cells and tumours, addressing their expression levels and comparing their transcriptional activities.

\section{Materials and methods}

Additional sequences used to analyse TFAP2A isoform conservation

Xenopus tropicalis (1a: NM_001037258.1, 1b: BC135698, 1c: CR848274.2); Danio rerio (1a: NM_176859.2, 1b: BC066582, 1c: AF457192.1).

\section{Antibodies and plasmids}

AP-2 $\alpha$ isoform-specific antibodies were generated utilising the peptides representing the different isoforms (1a: KLTDNIKYEDCEDRHDGTSN, 1b: LVHSFSAMDRH DC, 1c: SILAKMGDWQDRC 1d: GGARGQTGPGSAC), coupled to the KLH carrier protein, injected in rabbits according to standard protocols. The specificity of the antibodies was tested in Western blot on extracts of HepG2 overexpressing the different isoforms. Isoform 4 serum was specific and was further used in a dilution $1: 100$. Other isoform-specific antibodies were further purified utilising the peptides KLTDNIKYEDCEDRC (1a), LVHSFSAMDRC (1b), and SILAKMGDWQDRC (1c) coupled to a SulfoLink Coupling Gel Column (Thermo Fisher Scientific Inc, Rockford, IL, USA)), according to the manufacturer's instructions, eluted with the corresponding peptides, and concentrated. The isoform 1a antibody was further incubated on a SulfoLink column conjugated to the peptide (DRHDGTSNGTARLC) to retain exon 2 binding antibodies. Pan-AP-2 $\alpha$ (3B5), actin and HSC70 antibodies were from Santa Cruz Biotechnology Inc. (Santa Cruz, CA, USA). pcDNA3 encoding full length human AP- $2 \alpha$ isoform 1a [19] was mutagenised to the WT sequence (V2L) using the GeneEditor kit (Promega UK, Southampton, UK). Expression plasmids for isoforms 1b, 1c and $1 \mathrm{~d}$ were generated in the same background. The $\mathrm{N}$ terminal portion of the cDNA encoding AP- $2 \alpha$ isoform 1c was generated by PCR with the primers GATGTCCATACTTGCCAAAATGG and TGAGGTACATTTTGTCCATGGC from cDNA generated from MCF10A cells and sublcloned by KpnI-BlpI digestion into pcDNA3 AP- $2 \alpha$, thus substituting the 5 ' sequence of isoform 1a. pcDNA3 AP- $2 \alpha$ isoform $1 \mathrm{~b}$ was generated by substituting the HindIII-BlpI fragment of pcDNA3-AP-2 $\alpha-1$ a with the EarI-BlpI fragment of IMAGE clone 4432023. pcDNA3 AP- $2 \alpha$ isoform $1 d$ was generated by substituting the fragment HindIII-BlpI of pcDNA3-AP-2 $\alpha-1$ a with the SfoI-BlpI fragment from IMAGE clone OE37H07. All constructs were verified by sequencing. Isoform 1a was further mutagenised (K10R) using the QuickChange kit (Agilent Technologies UK Ltd, Wokingham, UK). Other plasmids were: pCI-p300 [20], pRc/CMV CBP [21], pcDNA3-CITED2 [11], pcDNA3-CITED4 [10], pSG5 sumo1 $\triangle \mathrm{HSTV}$ and pSG5 sumo2 $\triangle \mathrm{VY}$ generated by PCR, pSG5 ubc9 [15], pSG5 Ubc9 C93S [15]. Reporter plasmids were: 3xAP2-Bluc [11], p500LUC, generated by subcloning the $-500 /+40$ $E R B B 2$ promoter fragment from p500CAT [22] into pGL3-basic (Promega), and cyclin D3 generated by cloning the region $(-201,+162)$ into pGL3 basic. For normalisation, reporters expressing Renilla luciferase (pGL4.74 hRluc/TK or phRG-TK; Promega) were utilised.

\section{Cell culture, transfection and cell extracts}

All cell lines were from the ATCC and were cultivated according to the criteria provided: HepG2 cells DMEM supplemented with $10 \%$ foetal calf serum, penicillin and streptomycin; MCF10A - DMEM:F12 1:1 supplemented with EGF, hydrocortisone, cholera toxin, insulin, 5\% horse serum, penicillin and streptomycin [23]. Tamoxifen-resistant cell lines were cultivated in $10^{-7} \mathrm{M}$ tamoxifen for six months. Cells were transiently transfected with GeneJuice (Merck KGaA, Darmstadt, Germany) in a 24-well format for luciferase experiments $(80,000$ cells/well) or 6 -well format for Western blot 
experiments $(350,000$ cells/well) using a 1:3 ratio DNA: Genejuice. The total amount of DNA transfected was normalised using the appropriate empty vectors. Cells were harvested after $48 \mathrm{~h}$, lysed and assayed using the dual luciferase reporter system (Promega). Firefly luciferase readings were normalised to renilla luciferase values. All transfection experiments were performed in triplicate and repeated at least three times; the average, with the SE, is shown. Statistical analysis was performed utilising Student's $t$-test. Extracts for Western blot were generated utilising RIPA buffer $(50 \mathrm{mM}$ Tris- $\mathrm{HCl} \mathrm{pH}$ $7.5,150 \mathrm{mM} \mathrm{NaCl}, 0.5 \%$ sodium deoxycholate, $0.1 \%$ SDS, 1\% Nonidet P-40). Where indicated, isopeptidase inhibitors were included at $10 \mathrm{mM}$ iodoacetamide (IAA) and $20 \mathrm{mM}$ N-ethylmaleimide (NEM). Alternatively, cell pellets were resuspended directly in urea buffer $(8 \mathrm{M}$ urea, $1 \mathrm{M}$ thiourea, 0.5\% CHAPS, $50 \mathrm{mM}$ DTT, $24 \mathrm{mM}$ spermine). Western blot quantifications were achieved using ImageJ software [24], using a $t$-test for statistical analysis.

\section{RNA preparation and RT-PCR}

RNA was extracted using the RNAeasy kit (Qiagen UK, Crawley, UK). We used $1 \mu \mathrm{g}$ of total RNA to reverse transcribe with Superscript III (Life Technologies Corporation, Carlsbad, CA, USA). Real-time PCR of the different isoforms was performed using SybrGreen (Life Technologies) according to standard cycling protocols with $300 \mathrm{nM}$ of specific forward primer (isoform 1a: ATATCAAGTACGAGGACTGCG, isoform 1b: AGATGTTAGTTCACAGTTTTTCAGC, isoform 1c: GATGTCCATACTTGCCAAAATGG, isoform 1d: CCAAGCAGCTCCTACC), and $50 \mathrm{nM}$ of common reverse primer TTGCGACTGGGGGTAGATAG. Absolute amounts of each isoform were determined by generating a standard curve from a known amount of each linearised pcDNA3-AP-2 $\alpha$ plasmid.

\section{Tumour samples}

Fresh frozen tissue from $11 \mathrm{ER}+$ ve patients was obtained from Guys and St Thomas/King's College London (GSTFT/KCL) Breast Tissue Bank (Ethics approval number LREC Ref 06/Q0603/25). All were treated with tamoxifen after surgery; tamoxifen resistant patients had relapsed within two years. Normal breast tissue samples were provided by Prof. Louise Jones (LREC Ref 05Q403/ 199). Statistical analysis was performed using the MannWhitney test.

\section{Results}

TFAP2A alternative first exons are conserved in vertebrates

The human TFAP2A gene structure and expressed sequence tags (ESTs) were analysed and the existence of three isoforms, assigned as reference sequences named isoform 1a, 1b, and 1c, was confirmed (Figure 1a, b). In addition, the existence of a fourth isoform, whose exon is located between exons $1 \mathrm{a}$ and $1 \mathrm{~b}$ was suggested (Figure 1a). AP- $2 \alpha$ isoform 1a corresponds to the AP$2 \alpha$ cDNA originally cloned [19] and is represented by nine ESTs. AP- $2 \alpha$ isoforms $1 \mathrm{~b}$ and $1 \mathrm{c}$ are represented respectively by 10 and 8 ESTs. The fourth splice variant, which we named isoform $1 \mathrm{~d}$, is represented by three ESTs, only one of which, derived from a corneal cDNA library, is correctly spliced.

Alternative transcripts are not always functional, but a criterion that suggests biological activity is conservation across species. Our analysis revealed that highly conserved homologs of AP- $2 \alpha 1 \mathrm{a}$ and $1 \mathrm{~b}$ are found in mammals and in Xenopus tropicalis and Danio rerio. AP- $2 \alpha 1 \mathrm{c}$ is also well conserved in mammals, and has a homolog in $X$. tropicalis and $D$. rerio, although with a lower sequence conservation (Figure 1c). This conservation of the isoforms across species suggests they have been under positive selective pressure and hence have distinct roles required for normal development. The one exception may be $1 \mathrm{~d}$ since the sequence and structure of this exon is conserved only in primates; therefore, given that this isoform is represented by only one correctly spliced EST, its existence is more questionable.

All the alternative first exons identified encode for at least one possible initiator methionine. Exon 1a, 1b and 1c each encode for a very short sequence of amino acids, resulting in proteins with a very similar predicted molecular weight, while exon $1 \mathrm{~d}$ encodes for a longer $\mathrm{N}$-terminus (60 residues) (Figure $1 \mathrm{~b}$ ). All the reported isoforms - with the exception of $1 \mathrm{~d}$ - have two in-frame alternative methionine residues (Figure 1b). For isoform $1 \mathrm{a}$, the starting methionine was determined to be the downstream one by amino-terminal peptide sequencing when the protein was originally purified [19]. For isoforms $1 \mathrm{~b}$ and $1 \mathrm{c}$, since both methionine residues are conserved across different species, we searched for a Kozak sequence to help predict the relative strength of each translation initiation site [25]. This suggested that for isoform 1b, the downstream ATG may encode the predominant starting methionine residue. However, we could not differentiate between the two ATGs in isoform 1c since both have a conserved purine at position $-3$.

\section{TFAP2A isoforms $1 \mathrm{a}$ and $1 \mathrm{c}$ are expressed at a similar level in breast cell lines and tissue}

In order to examine the relative expression of the different isoforms in breast tissue, a specific real time PCR assay was set up to discriminate between each of the amino-terminal variants. This analysis revealed that isoform 1a is the predominant isoform at the RNA level in 


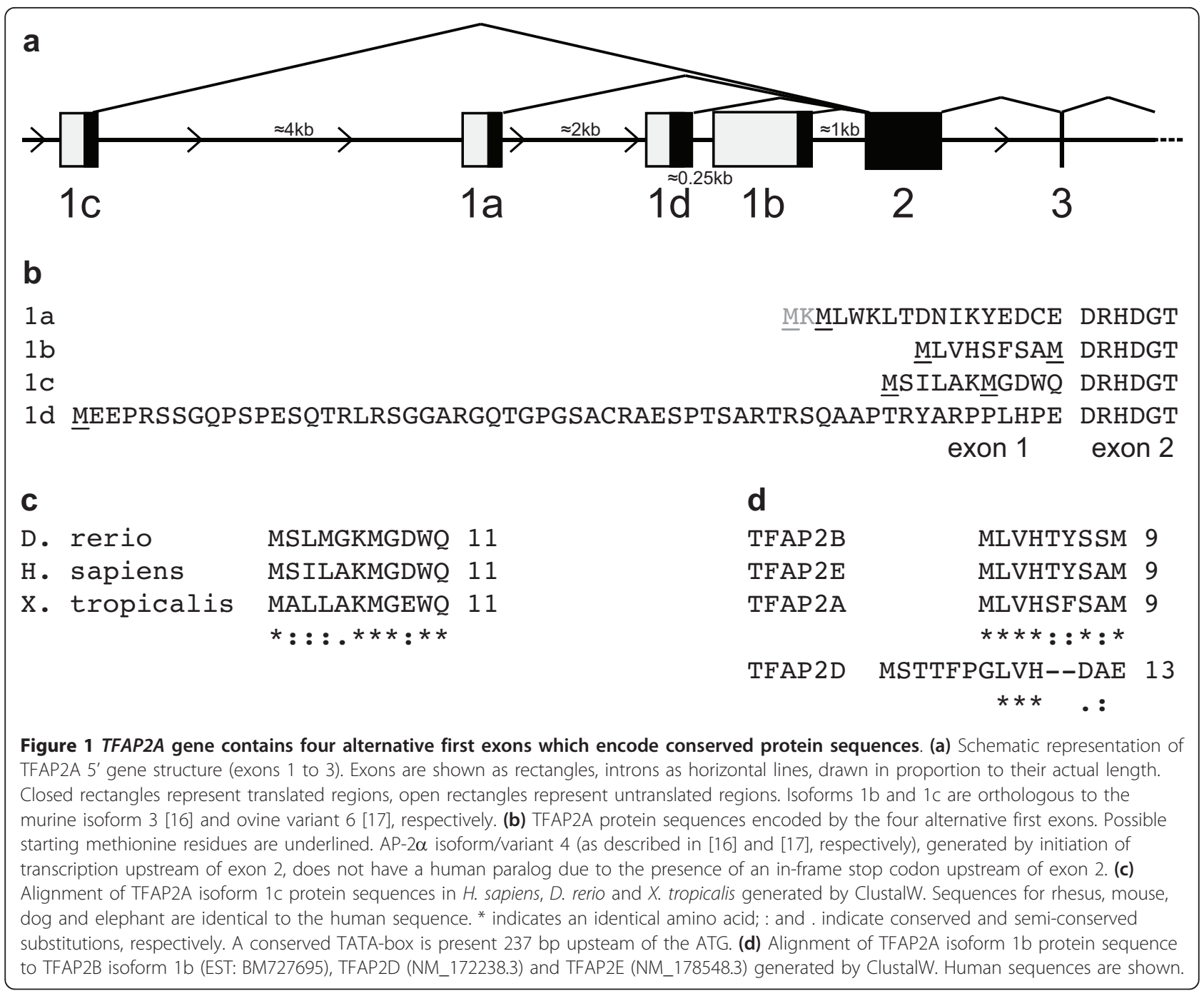

a panel of different breast cell lines and four samples of normal breast tissue (Figure 2a). A significant, albeit lower, expression of isoforms $1 \mathrm{~b}$ and $1 \mathrm{c}$ was also detected in the cell lines and the tissue samples (Figure $2 a)$. In contrast, isoform $1 \mathrm{~d}$ levels were very low, suggesting that this isoform, if it exists, does not play a prominent role in the breast. $1 \mathrm{~d}$ expression was investigated additionally in an RNA sample from human foetal eye where, again, the mRNA levels were undetectable (not shown).

To investigate expression at the protein level, isoform specific antibodies were generated (as described in Materials and methods). An antibody affinity analysis was performed by transfecting HepG2 cells, which do not express detectable levels of AP- $2 \alpha$, with specific expression plasmids generated for each isoform, under the control of the CMV promoter. The expression levels of the different isoforms, as assessed using an antibody against the common DNA binding domain of the protein (pan-AP-2 $\alpha, 3 B 5$ ), were consistently similar (Figure $2 \mathrm{~b}$ ). The same quantity of each lysate was used as a loading control for subsequent isoform-specific Western blots (Figure 2b). AP-2 $\alpha$ 1a and $1 \mathrm{c}$ antibodies bound to their respective isoform with high affinity, while the $1 \mathrm{~b}$ specific antibody showed significantly lower affinity (compare Figures $2 \mathrm{~b}$ and 2c). Western blot analysis of a panel of breast tumour lines showed that isoform 1a was expressed at modest levels in all the cell lines investigated (Figure 2c). A faint band corresponding to the molecular weight of isoform $1 \mathrm{~b}$ could be detected in all the cell lines, but the low affinity of the antibody made it difficult to reliably distinguish it above the background (Figure 2c). In contrast, isoform 1c was expressed at significant levels in all the cell lines investigated, with the exception of Cal51, which also lacked detectable expression at the mRNA level (Figure 2a, c). Isoform 1d protein was not detected in any of the cell lines (not shown), in accordance with the mRNA data. 


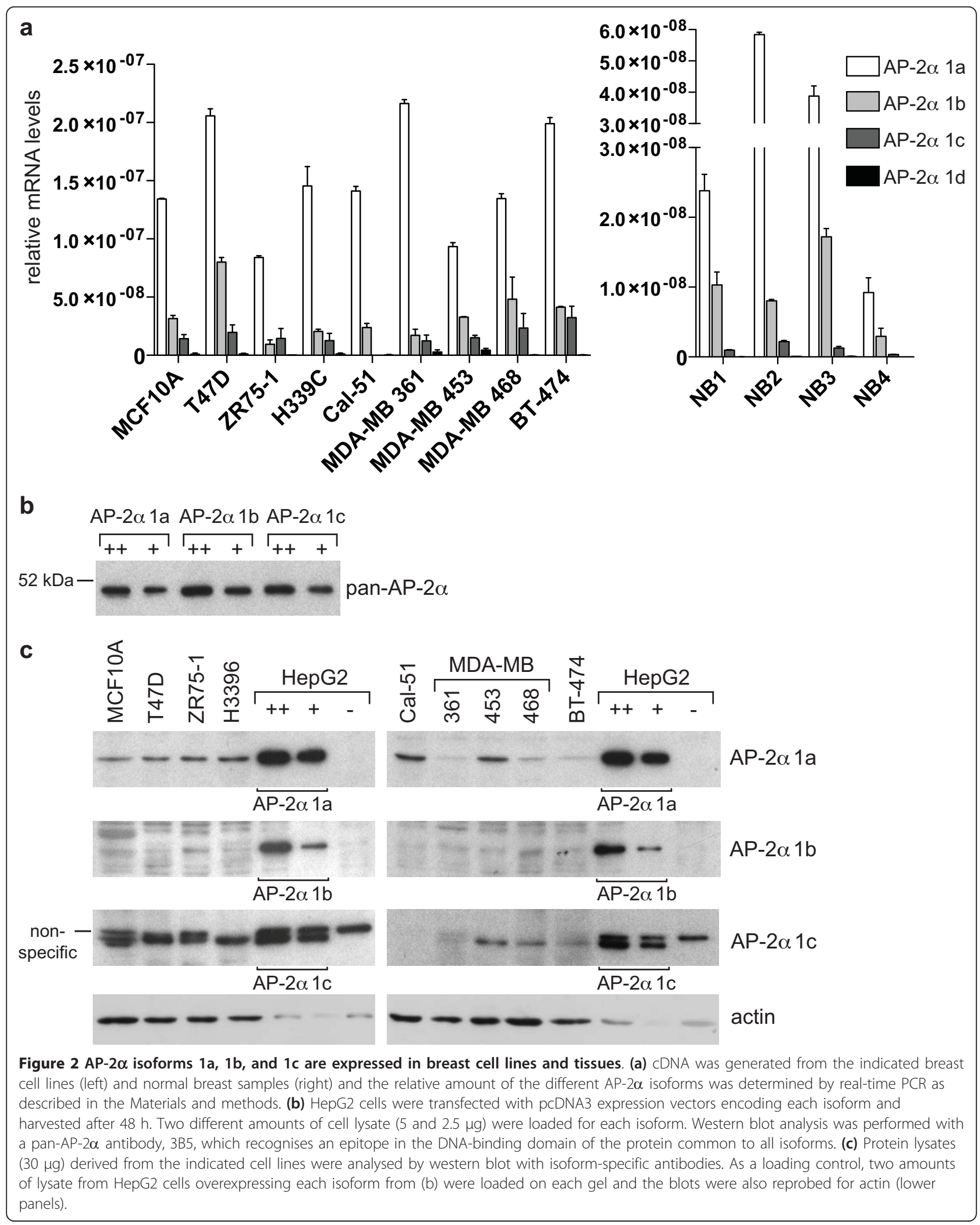


Comparing the intensity of signal between the breast line and transfected HepG2 control lysates, suggested that protein levels of isoform $1 \mathrm{c}$ are at least comparable to those of isoform 1a in many lines. This was examined graphically, by scanning the Western blots (see Figure S1 in Additional File 1), which demonstrated that isoform $1 \mathrm{c}$ is expressed at significantly higher levels in the majority of lines examined, including MCF10A, T47D, ZR75-1, MDA-MB 361 and MDA-MB 468 cells. Therefore, the protein levels of isoforms $1 \mathrm{a}$ and $1 \mathrm{c}$ are much more similar than would be inferred from the mRNA data alone and this disproportion between mRNA and protein levels suggests that these two isoforms are differentially regulated either at the translational or posttranslational level.

\section{TFAP2A isoforms share a similar transactivation mechanism}

We next explored whether the isoforms have distinct biological properties. The DNA binding activity of the isoforms was compared using electromobility shift assays (EMSA) and nuclear extracts from HepG2 cells transfected for each of the different isoforms. This revealed that they all have a similar affinity for a consensus AP-2 DNA binding site (Figure S2 in Additional File 1). All the isoforms showed a high stability, without detectable differences in half-life when exogenously expressed in HepG2 cells (not shown).

To determine whether all the isoforms can act as transactivators in combination with CITED2 or 4 and CBP or p300, their activity was analysed in HepG2 cells cotransfected with a synthetic AP-2 dependent luciferase reporter construct widely used to characterise AP-2 activity [11]. Most expression constructs for the 1 a isoform carry a mutation at the second amino acid (V2L), introduced when AP-2 $\alpha$ was first cloned [19], which we mutated back to the wild type sequence in order to accurately compare the isoforms. All showed similar basal levels of transactivation activity (Figure 3, open boxes). When cotransfected with the adaptors CITED2 or CITED4, all isoforms gave enhanced activation of the reporter $(P<0.05)$ compared to the control (Figure 3, grey and black boxes). Cotransfection with p300 or CBP in combination with the different CITED factors resulted in a further increase in transactivation activity $(P<0.05)$ for isoforms $1 \mathrm{a}, 1 \mathrm{~b}$, and $1 \mathrm{c}$ (Figure 3$)$. An exception was isoform $1 \mathrm{~d}$, which did not further transactivate the reporter in the presence of CBP. Moreover, transactivation levels for this isoform were lower compared with the others. Taken together, these data suggest that all isoforms (excluding 1d) are able to interact functionally with the adaptors CITED2 or CITED4 with similar efficiency leading to the recruitment of p300 or CBP.

\section{TFAP2A 1a represses the cyclin D3 promoter via a sumo-} dependent mechanism

Transcriptional activity of the isoforms was further compared using natural promoters from genes known to be regulated by $\mathrm{AP}-2 \alpha$, including those shown to be repressed by AP- 2 factors. We have observed that AP$2 \alpha$ downregulates cyclin D3 expression, through direct binding to sites within the proximal promoter (KV Chan and HC Hurst, unpublished). The activity of AP$2 \alpha$ isoforms on the cyclin D3 promoter was compared in HepG2 cells transfected with a reporter comprising the minimal promoter region $(-201 /+162)$ able to drive cyclin D3 expression. Isoforms $1 \mathrm{~b}$ and $1 \mathrm{c}$ failed to exert

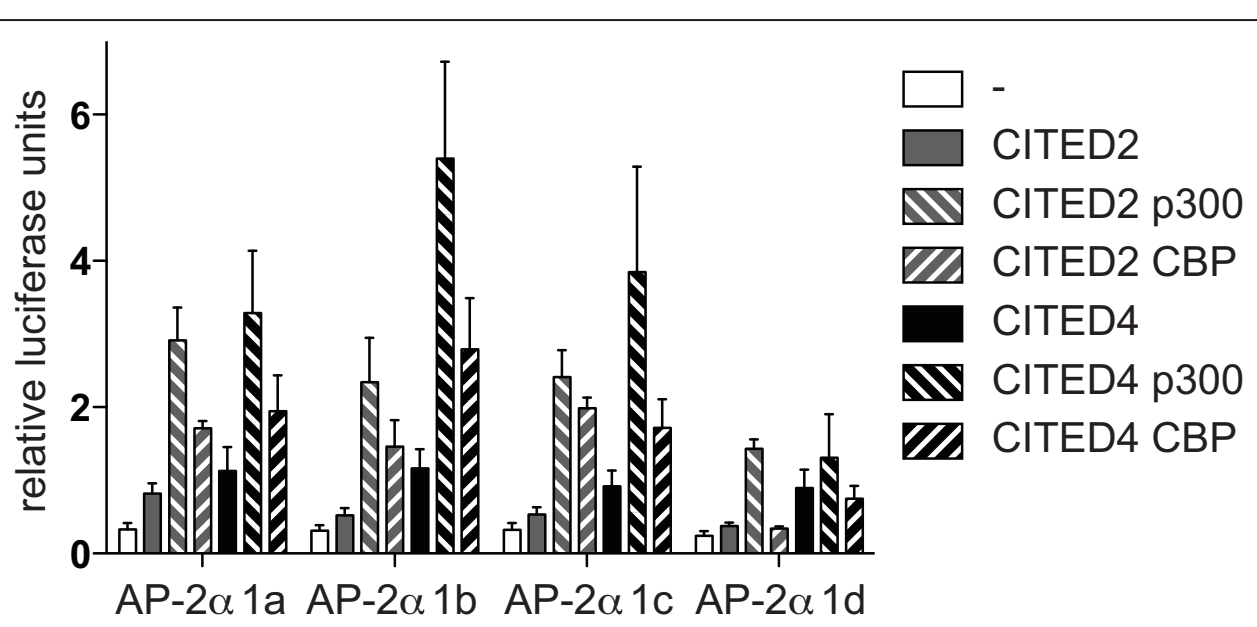

Figure 3 AP-2 $\alpha$ isoforms exert similar transactivation activity when cotransfected with CITED2 or 4 and p300 or CBP. HepG2 cells were transfected with $0.05 \mu \mathrm{g}$ pcDNA3-AP-2 $\alpha, 0.25 \mu \mathrm{g}$ 3xAP2-Bluc, $0.25 \mu \mathrm{g}$ phRG-renilla, $0.25 \mu \mathrm{g}$ pcDNA3-CITED2/4, $1 \mu \mathrm{g}$ pCI-p300 or pRc/CMV CBP as indicated. Relative firefly luciferase activity normalised to renilla luciferase activity is shown. Average and standard error from four independent experiments is reported. 
any effect on the cyclin D3 promoter at any ratio of reporter: expression construct tested (Figure 4). In contrast, isoform 1a had a reproducible and significant repressive effect (Figure 4). Isoform 1d also exerted a very pronounced inhibitory effect, up to 50\% (Figure 4). The inhibitory effect at a 1:1 ratio was already maximal for isoforms $1 \mathrm{a}$ and $1 \mathrm{~d}$, and did not change at higher ratios for isoforms $1 \mathrm{~b}$ or $1 \mathrm{c}$ (not shown).

A key difference between AP- $2 \alpha$ isoform $1 \mathrm{a}$ and the other two isoforms expressed in breast is the presence of a putative sumoylation site (IKYE) centred at lysine 10 within the sequence encoded by exon $1 \mathrm{a}$. This site is homologous to the site found in AP- $2 \gamma$ which has previously been demonstrated to be conjugated to SUMO in vivo thereby reducing AP- $2 \gamma$ transactivation activity [15]. Consequently, we hypothesised that this post-transcriptional modification may also play a role in the negative regulation of the cyclin D3 promoter by AP- $2 \alpha$ isoform 1a. To test this, a non-sumolylalable mutant of isoform 1a, K10R, was generated and found to lack inhibitory activity on the cyclin D3 reporter construct (Figure 4). AP-2 $\alpha$ has previously been demonstrated to interact with the SUMO E2-conjugating enzyme, UBC9, in a yeast two-hybrid screen [15], but to confirm that AP-2 $\alpha$ can be sumoylated in vivo, expression constructs for UBC9 and SUMO-1 (Figure 5a) or SUMO-2 (not shown) were cotransfected in HepG2 cells together with the different AP- $2 \alpha$ isoforms. This resulted in the appearance, for isoform 1a only, of a slower migrating band at approximately $75 \mathrm{kDa}$, compatible with a mono-sumoylated form of AP- $2 \alpha$ (Figure $5 \mathrm{a}$ ). Furthermore, mutation of lysine 10 led to a very pronounced reduction in intensity of this sumoylated band, thus confirming that lysine 10 is the predominant sumoylation site in isoform 1a, and explaining why the other isoforms were not significantly modified.

To confirm that the inhibitory activity of isoform 1a is dependent on sumoylation, HepG2 cells were cotransfected with the 3xAP2-Bluc reporter and increasing doses of SUMO-1 or SUMO-2. The transactivation activity was reduced with SUMO co-transfection, particularly by SUMO-2, while the transactivation activity of the K10R mutant or AP-2 $\alpha$ 1c was not altered significantly, suggesting that the most important sumoylation site is indeed lysine 10 of isoform 1a (Figure 5b). To confirm that the inhibitory effect exerted by isoform 1a on the cyclin D3 reporter is due to sumoylation, we co-transfected with either wild-type UBC9 or its sumoylation defective mutant, C93S [26]. While transfection of wt UBC9 did not result in further inhibition of cyclin D3 reporter activity, cotransfection of UBC9-C93S partially reverted the inhibitory activity of isoform 1a (Figure 5c). In addition, a clear reduction in the level of sumoylated AP- $2 \alpha$ was observed by western blot when the $1 \mathrm{a}$ isoform and UBC9-C93S were co-expressed in the same proportion in HepG2 cells (Figure S3 in Additional File 1).

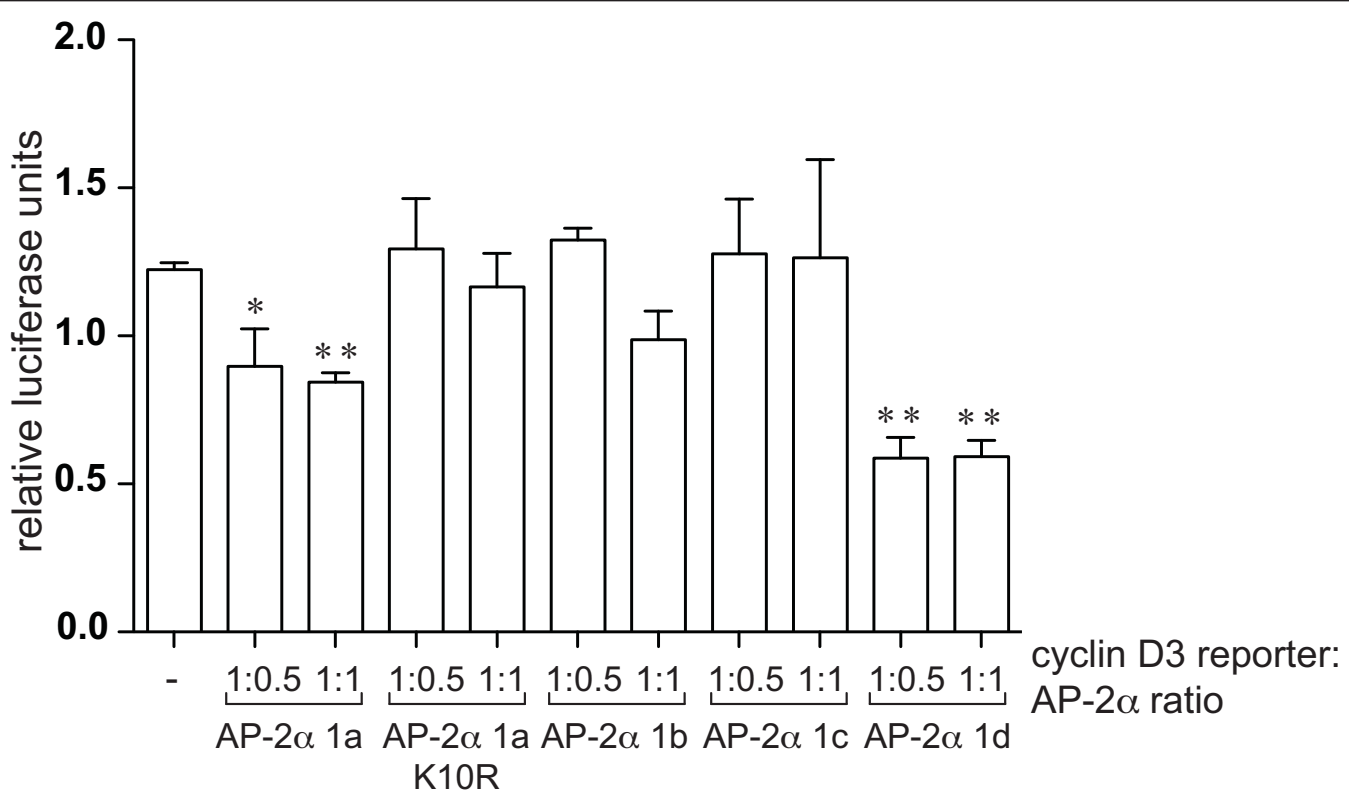

Figure 4 AP-2 $\alpha$ isoforms differ in their transcriptional repression activity. HepG2 cells were transfected with $0.25 \mu \mathrm{g} /$ well pGL4.74 (Renilla), $0.4 \mu \mathrm{g} /$ well cyclin D3 reporter construct, and the indicated ratios of pcDNA3-AP-2 $\alpha$ (corresponding to 0.2 and $0.4 \mu \mathrm{g} /$ well). Results are reported as relative firefly luciferase activity normalised to renilla luciferase activity. Average and standard error from three independent experiments is shown. 
a
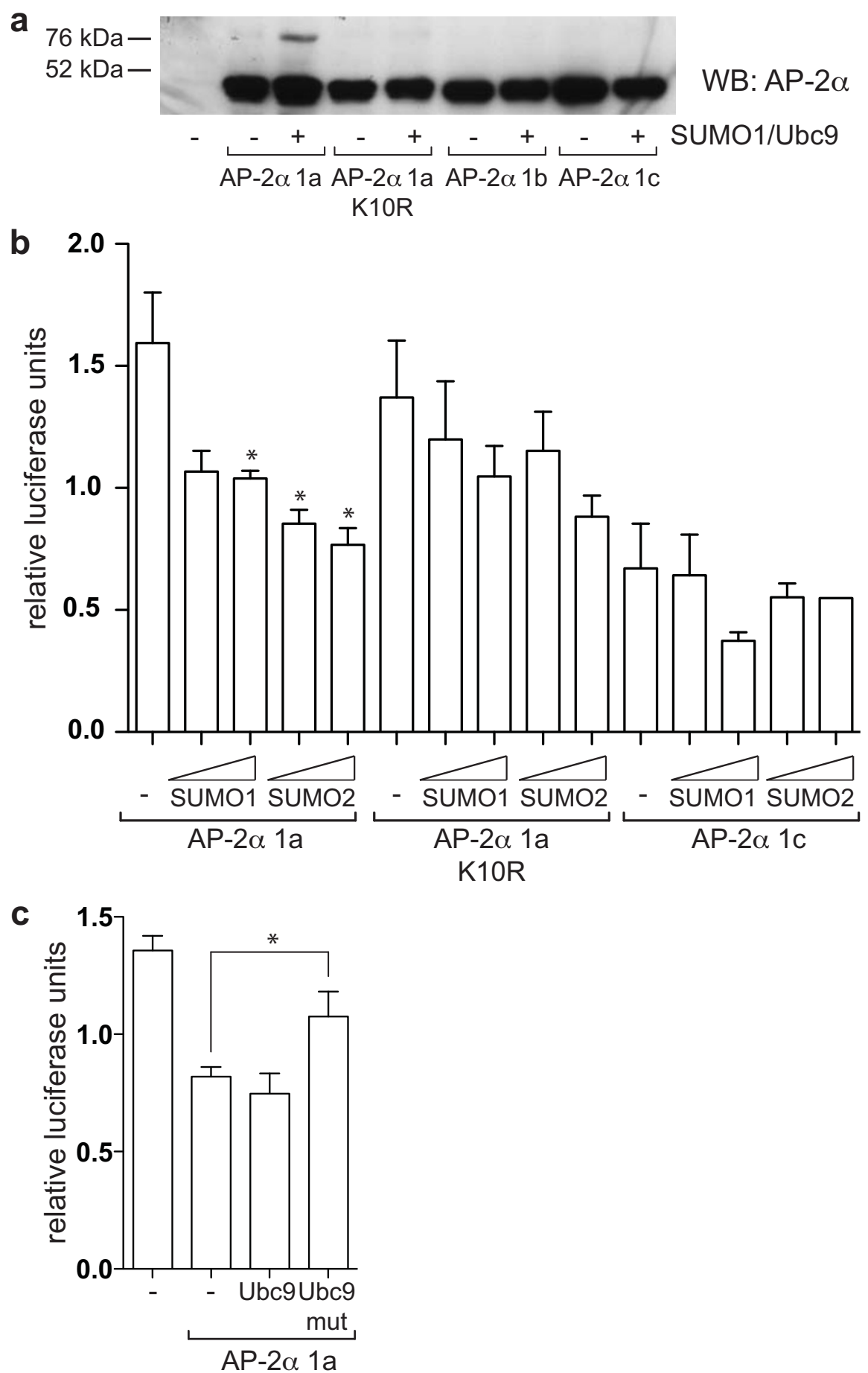

Figure 5 AP-2 $\alpha$ isoform 1a can be sumoylated leading to decreased transactivation activity. (a) HepG2 cells were transfected with $0.3 \mu \mathrm{g} /$ well of the different pcDNA3-AP-2 $\alpha$ constructs, without and with $0.1 \mu \mathrm{g} /$ well pSG5 Ubc9 and $0.6 \mu \mathrm{g} / \mathrm{well}$ pSG5 SUMO1 in a six-well format. $48 \mathrm{~h}$ after transfection, lysates were harvested in RIPA buffer containing IAA and NEM, and analysed by western blot with a pan AP-2 $\alpha$ antibody (3B5). One experiment representative of three is shown. A second, weaker sumoylation site (IKKG) was predicted (using "SUMOplot") within the Cterminal half of the protein which could explain the low levels of sumoylation observed for AP- $2 \alpha$ K10R and the other isoforms using longer exposures. (b) HepG2 cells were transfected with $0.05 \mu \mathrm{g} /$ well of the different pcDNA3-AP-2 $\alpha$ constructs, $0.25 \mu \mathrm{g} /$ well 3xAP2-Bluc, $0.25 \mu \mathrm{g} / \mathrm{well}$ phRG-renilla, $0.25 \mathrm{\mu g} /$ well pcDNA3-CITED2, $0.75 \mu \mathrm{g} /$ well pCl-p300, and 0.25 or $0.5 \mu \mathrm{g} /$ well pSG-SUMO1/2 as indicated. Relative firefly luciferase activity normalised to renilla luciferase activity is shown. The average and standard error of three experiments is reported. (c) HepG2 cells were transfected with $0.2 \mu \mathrm{g} /$ well pGL4.74 (Renilla), $0.3 \mu \mathrm{g} /$ well cyclin D3 reporter, $0.15 \mu \mathrm{g} /$ well pcDNA3-AP-2 $\alpha$ isoform 1a, $0.3 \mu \mathrm{g} /$ well pSG-Ubc9 and $0.15 \mu \mathrm{g} /$ well PSG-SUMO1. Average and standard error from three independent experiments is represented. 
TFAP2A isoform $1 \mathrm{a}$ is a weaker transactivator of the ERBB2 promoter

The transactivation activity of the different AP- $2 \alpha$ isoforms was further tested on the $E R B B 2$ promoter, which is known to possess two functional AP-2 binding sites, mapping at -210 and -500 upstream of the transcription start site, which are required for positive regulation of $E R B B 2$ by AP- $2 \alpha$ in breast tumour lines [22,27]. A reporter carrying $E R B B 2$ promoter sequences $(-500 /+40)$ was co-transfected at different ratios with the AP- $2 \alpha$ isoform expression constructs together with Cited2/p300 (Figure 6). Isoform 1a significantly induced reporter activity at a $1: 2$ and 1:4 reporter:AP- $2 \alpha$ ratio (Figure 6). In contrast, the transactivation activity of isoforms $1 \mathrm{~b}$ and $1 \mathrm{c}$ was already significant at a ratio of 1:0.5, and further increased at higher ratios, reaching a plateau at 1:4. Isoform $1 \mathrm{~d}$ was the most potent transactivator and its activity increased at higher ratios in an almost linear manner. Consequently, it appears that isoform 1a is the weakest transcriptional activator on the ERBB2 promoter, Thus, when tested in the context of a complex, natural promoter, differences in transactivation activity between the TFAP2A isoforms are observed which were not evident when using the artificial reporter construct (Figure 3). To examine this further, the relative activity of isoforms 1a, $1 \mathrm{~b}$ and $1 \mathrm{c}$ on the ERBB2 promoter was also compared in the presence of different combinations of the CITED/
p300/CBP cofactors (Figure S4 in Additional File 1). In each case, isoform 1a was the weakest activator.

\section{TFAP2A isoform 1c expression increases in tamoxifen-} resistant lines and tumours

Since we observed that AP- $2 \alpha$ isoforms have differential transactivation activity on the $E R B B 2$ promoter, we decided to investigate their expression in a biological context. ErbB2 overexpression is associated with resistance to the oestrogen receptor (ER) antagonist tamoxifen, since signalling from the receptor can promote oestrogen independent activation of the ER (reviewed [28]). We, therefore, investigated whether AP- $2 \alpha$ isoform levels were altered in tamoxifen resistant (TR) lines and tumour samples. Given that isoform 1a was the weakest activator of ERBB2 expression (Figure 6), we would predict that increased expression of the other isoforms might be associated with the tamoxifen resistant phenotype. In three independent TR lines, which were cultivated in the presence of tamoxifen for at least six months, we observed a pronounced increase in levels of ErbB2 compared to the wt controls (Figure S5a, b in Additional File 1). In the same three lines, levels of isoform 1c compared to isoform 1a increased significantly, as measured by quantifying levels in Western blots as the ratio between the two isoforms (1c:1a; $P<0.05$, see Figure $7 \mathrm{a}, \mathrm{b})$.

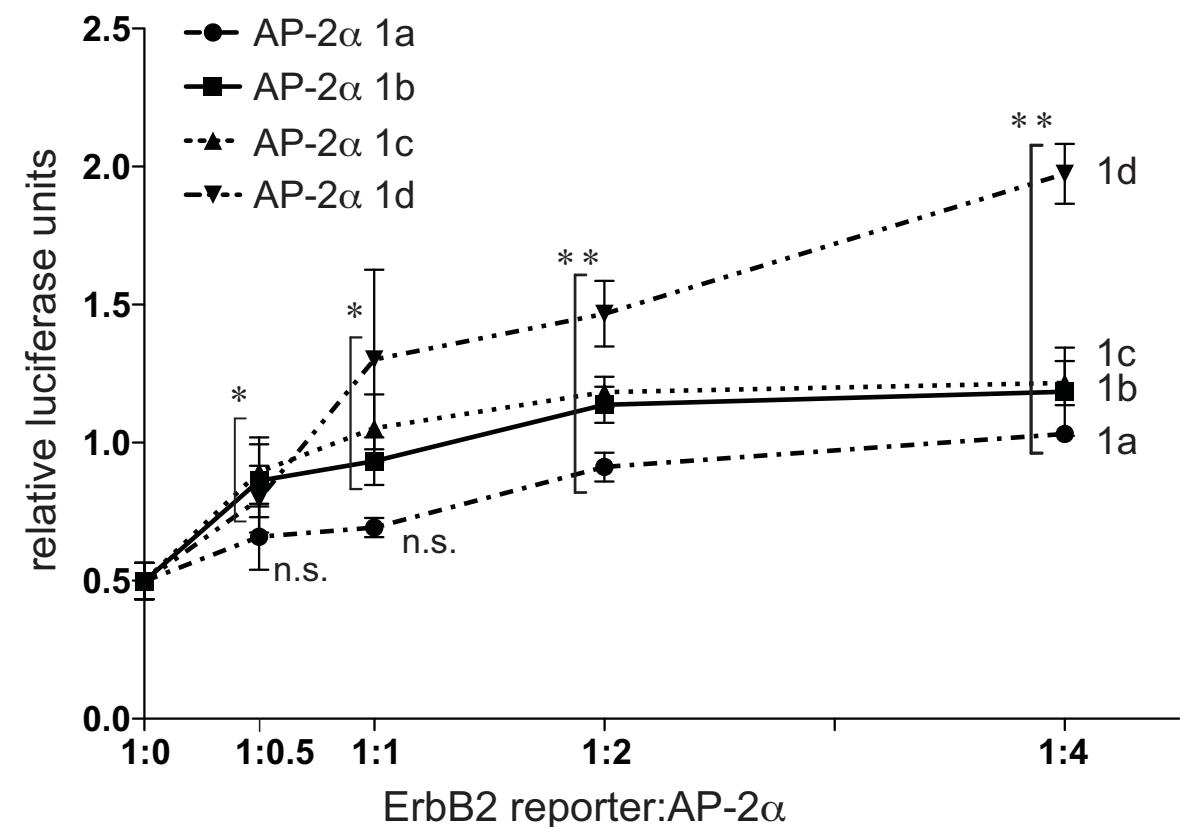

Figure 6 AP-2 $\alpha$ isoforms exert different levels of transactivation activity at the ERBB2 promoter. HepG2 cells were transfected with 0.12 $\mu \mathrm{g} /$ well p500 ErbB2-luc, $0.02 \mu \mathrm{g} /$ well renilla hRG, $0.06 \mu \mathrm{g} /$ well pCI-p300, $0.06 \mu \mathrm{g} /$ well pcDNA3-CITED2, and indicated ratios (corresponding to a total of $0.06,0.12,0.24$ and $0.48 \mu \mathrm{g} /$ well) of the different pcDNA3-AP- $2 \alpha$ constructs. The average and standard error of three independent experiments is represented. 
a

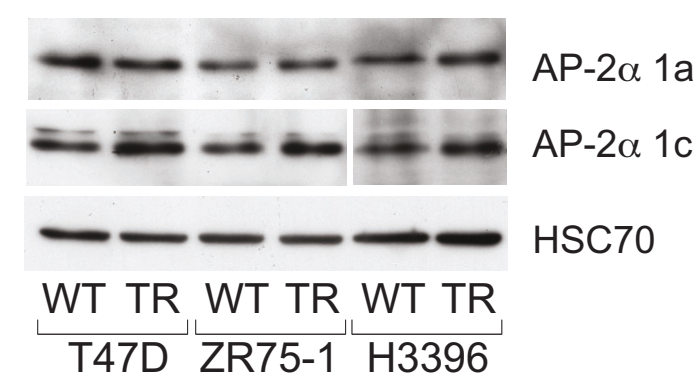

C

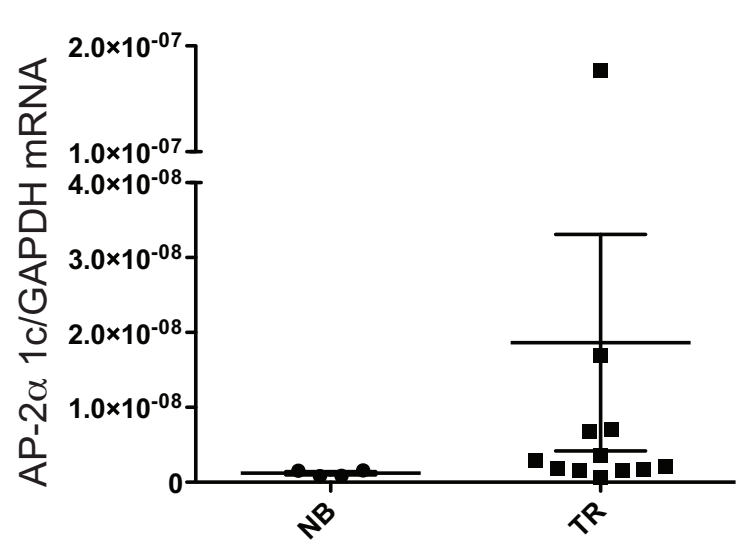

b

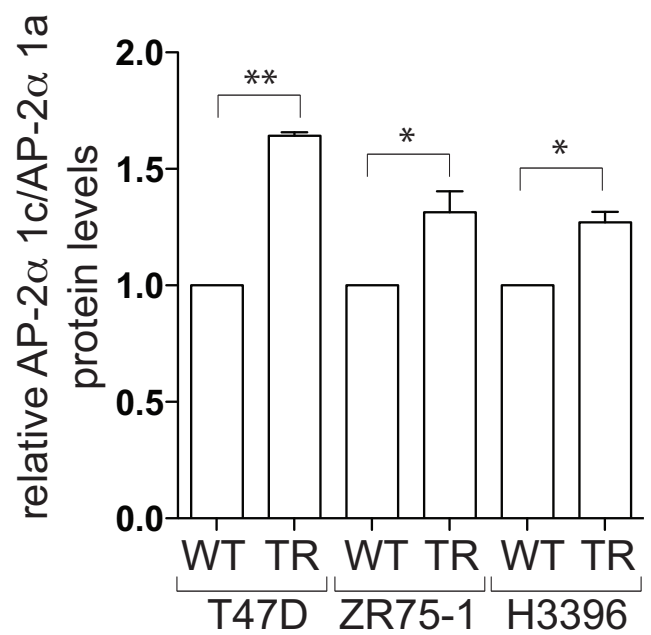

d

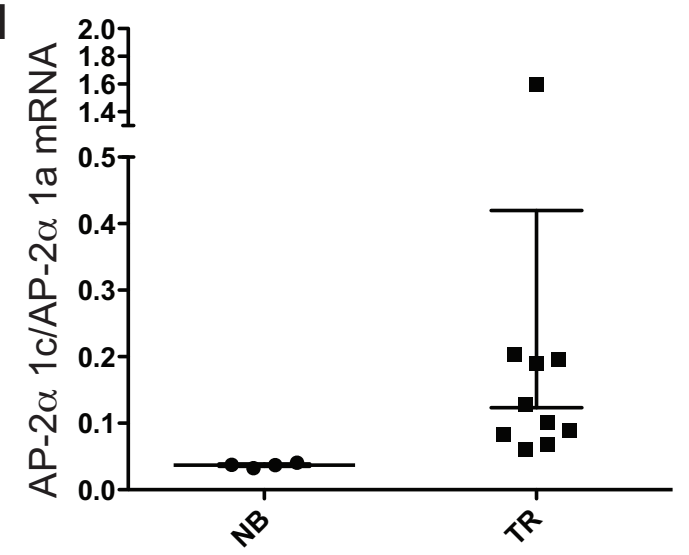

Figure 7 AP-2 $\alpha$ isoform expression is differentially regulated in tamoxifen resistant cell lines and breast tumour samples. (a, b): Levels of AP- $2 \alpha$ isoform 1a and 1c were determined by Western blot in a series of wild-type ER+ breast tumour lines and in their tamoxifen-resistant counterparts. The signal from a number of different non-saturated exposures was quantified with ImageJ and is graphically represented in (b). (c, d): Normal breast (4) and tumour samples (10) were analysed by real-time PCR for AP-2 $\alpha$ isoform expression levels. The relative levels of AP-2 $\alpha$ $1 \mathrm{c}$ normalised using GAPDH levels (c) and the ratio between AP-2 $\alpha$ 1c and 1a (d) are represented. The differences remain statistically significant even when the outliers are eliminated from the analysis.

This finding led us to compare TFAP2A isoform expression levels in a small series of mRNA samples from TR tumours. Good quality RNA from frozen samples was required to detect isoforms levels with sufficient sensitivity, and this limited the number of samples available. In these samples, ERBB2 mRNA levels tended to be higher compared to normal breast samples (Figure S3c in Additional File $1 ; P=0.043$ ). AP- $2 \alpha$ isoform $1 c$ levels were also higher in TR tumours compared to normal breast (Figure $7 \mathrm{c}, P=0.038)$. No significant change was observed for isoform 1a or 1b. Moreover, the difference in ratio between levels of isoform 1c and 1a in the TR tumours compared with normal breast was also significant (Figure $7 \mathrm{~d}, P=$ 0.002). This would suggest a selective and differential regulation of AP- $2 \alpha$ isoforms in tamoxifen resistant tumours.

\section{Discussion}

A significant proportion of the reports investigating the biological function of AP- $2 \alpha$ are overexpression studies which analyse exclusively the first isoform (1a) cloned. The existence of additional TFAP2A transcripts deriving from alternative first exons has been described in some mammals, but there has been little attempt to determine their importance or function. Our in silico analysis confirmed that different isoforms of AP- $2 \alpha$ occur in humans, providing at the same time evidence of a strong conservation throughout vertebrate evolution (Figure 1). Furthermore, ESTs for isoforms $1 \mathrm{a}$ and $1 \mathrm{~b}$ can also be found for the paralogous gene AP- $2 \beta$. In contrast, we were not able to identify alternative $5^{\prime}$ variants for AP$2 \gamma$, which is the homolog of AP- $2 \alpha$ isoform $1 \mathrm{a}$. 
Interestingly, AP $-2 \varepsilon$, and possibly the more distantly related $\mathrm{AP}-2 \delta$, are more similar to isoform 1b (Figure $1 \mathrm{~d})$, suggesting this is the most ancient isoform from which the family originated in vertebrates. Since we were unable to detect expression of isoform $1 \mathrm{~d}$ in the tissues examined, it probably does not represent a true isoform. However, the remaining AP- $2 \alpha$ isoforms are expressed at significant levels in breast cell lines and tissue (Figure 2) with the AP-2 $\alpha$ 1c protein being found at levels at least comparable to those of the initially identified isoform 1a, suggesting that its activity in the breast should be investigated.

Our analysis focused on testing whether differences in amino-terminal sequence lead to distinct biological characteristics for the AP- $2 \alpha$ isoforms. The N-terminus of AP- $2 \alpha$ encompasses the transactivation domain, and is not involved either in DNA binding or in protein dimerisation [1]. Therefore, functional differences are more likely to be found in transactivation activity, although the limited difference in sequence might suggest subtle variations. With a synthetic reporter, isoforms $1 \mathrm{a}, 1 \mathrm{~b}$ and $1 \mathrm{c}$ showed similar transactivation activity indicating a similar ability to interact functionally with CITED2 or CITED4 and p300 or CBP. This agrees with the finding that the domain essential for interaction with CITED2 lies in the central (common) region of AP-2 $\alpha$ [9]. However, the picture changed when the transactivation activity of the isoforms was compared using natural promoters. The cyclin D3 promoter was differentially regulated by the different isoforms with AP- $2 \alpha 1 \mathrm{~b}$ and $1 \mathrm{c}$ having a minimal effect, whereas isoform 1a exerted a significant inhibitory activity, similar to the effect exerted by AP- $2 \gamma$ (KV Chan and HC Hurst, unpublished). Lysine 10, which lies within a putative sumoylation motif, was essential for this inhibitory activity. An interaction between AP- $2 \alpha$ and UBC9 has been demonstrated previously [15] and our subsequent experiments showed that isoform 1a alone could be sumoylated in HepG2 cells. However, sumoylation of endogenous AP- $2 \alpha$ in breast lines could not be confirmed. When a similar experiment was performed for AP- $2 \gamma$ using MCF7 cells, which express it at high levels, only a small fraction of the protein was found to be sumoylated [15], in accord with similar studies on other sumoylated proteins [29]. By extrapolation, since in breast lines levels of isoform 1a represent only a proportion of the total AP- $2 \alpha$ protein (Figure 2), it is likely that the fraction of sumoylated isoform 1a falls below the level detectable using Western blotting or immunoprecipitation. Co-transfection of SUMO-1 or SUMO-2 with isoform 1a resulted in reduced transactivation activity, similar to observations made for AP- $2 \gamma$ [15]. The finding that for AP- $2 \alpha$ only isoform 1a can act as a repressor strengthens the hypothesis that sumoylation is necessary for AP-2 transcriptional repression. Moreover, we have linked for the first time the negative regulatory effect on the natural cyclin D3 promoter to sumoylation of AP- $2 \alpha$, since overexpression of mutant Ubc9 reverted the inhibitory activity of isoform $1 \mathrm{a}$.

On the ERBB2 promoter, isoforms $1 \mathrm{~b}$ and $1 \mathrm{c}$ both exerted significant transactivation activity at low reporter:expression plasmid ratios (Figure 6), while isoform 1a only achieved a similar level of transactivation activity when two to four times more expression plasmid was used, despite very similar expression efficiencies for all three expression constructs. This was initially explored using CITED2/p300 cofactors since CITED2 is considered to be the most biologically relevant cofactor for TFAP2A due to the similarity in phenotype between the respective knock-out mice. Moreover, a recent genomewide analysis of CBP and p300 binding suggested that p300 is significantly more associated with AP-2 sites compared to CBP [30]. However, we have also compared cofactor preferences for the TFAP2A isoforms on both the synthetic and $E R B B 2$ promoters at a variety of ratios (Figures 3, 6, S4 and data not shown) and noted that CITED4 also acts efficiently with isoforms $1 \mathrm{~b}$ and 1c. However, with all cofactor combinations, isoform 1a was consistently the weakest transactivator at the natural $E R B B 2$ promoter. It is significant, therefore, that we have observed that isoform 1c levels are higher in tamoxifen resistant breast tumour samples and cell lines. Although this observation has to be treated with some caution because of the limited number of samples available to assay, it suggests that AP- $2 \alpha$ isoforms may have differential roles in tumourigenesis due to variations in their transactivation activity on key target genes such as ERBB2.

These data demonstrate that the short sequence of amino acids encoded by the first exons affects significantly the transactivation potential of AP- $2 \alpha$. In particular isoform 1a may be a weaker transactivator and, potentially, has a more specialised function as an inhibitor of transcription on AP- $2 \alpha$ repressed genes. The lack of repressor activity shown by isoforms $1 \mathrm{~b}$ and $1 \mathrm{c}$ may be explained by the absence of the sumoylation motif. Alternatively, the sequence encoded by these isoforms may mediate an enhanced activation function that masks any inhibitory activity. Isoform 1c possesses a well conserved lysine residue, which is a possible target for a variety of regulatory post-translational modifications, including ubiquitination, acetylation and methylation, which will be the object of further investigations.

\section{Conclusions}

The different isoforms of AP- $2 \alpha$ possess differential transactivation and repression activities which are dependent on the promoter context. AP- $2 \alpha$ isoform 1c 
is expressed at significant levels in breast cell lines and tissues, and can be the predominant isoform. These observations underline the need to determine which isoforms are expressed at the protein level in different tissues and tumours, and that the complexity of the different AP- $2 \alpha$ isoforms has to be considered when conducting promoter studies on AP-2 target genes. Moreover, further studies are needed to analyse AP- $2 \alpha$ isoform 1c function in the breast and during tumourigenesis. Indeed, differences between the isoforms may help to reconcile contradictory effects reported for AP$2 \alpha$, in particular in breast tumour studies, and also may explain the partially overlapping yet distinct roles observed for AP- $2 \alpha, \beta$ and $\gamma$ during development.

\section{Additional material}

Additional file 1: Supplementary Figures S1 to S5. A PDF file with each of the supplementary figures referred to in the text, together with an explanatory legend.

\section{Abbreviations}

DCIS: ductal carcinoma in situ; EMSA: electromobility shift assay; EST: expressed sequence tag; IAA: iodoacetamide; NEM: N-ethylmaleimide.

\section{Acknowledgements}

The authors are grateful to Trevor Williams and lan Hart for their critical reading of the manuscript. We would like to thank Cheng Yeoh for RNA from tamoxifen resistant cell lines and tumours, and Sabine Gogolok and Mandy Tsang for their technical input into the project.

This work was supported by a Cancer Research UK programme grant (to $\mathrm{HCH}$ ) number C6775/A6250 and a Breast Cancer campaign grant (to AGS) number 2009MayPR60. KVC was supported by a Cancer Research UK studentship.

\section{Authors' contributions}

CB carried out the majority of the experiments and drafted the manuscript. KVC set up the cyclin D3 reporter assay. AMP set up the 3XAP-2 Bluc assay. $M C$ contributed to the isoform cloning and to the antibody purifications. AGS performed the EMSA analysis. $\mathrm{HCH}$ conceived the study, and participated in its design and coordination and helped to draft the manuscript. All authors read and approved the final manuscript.

\section{Competing interests}

The authors declare that they have no competing interests.

Received: 7 December 2010 Revised: 9 February 2011

Accepted: 4 March 2011 Published: 4 March 2011

\section{References}

1. Eckert D, Buhl S, Weber $S$, Jäger $R$, Schorle H: The AP-2 family of transcription factors. Genome Biol 2005, 6:246.

2. Friedrichs $N$, Jäger $R$, Paggen E, Rudlowski C, Merkelbach-Bruse S, Schorle H, Buettner R: Distinct spatial expression patterns of AP-2alpha and AP2gamma in non-neoplastic human breast and breast cancer. Mod Pathol 2005, 18:431-438.

3. Friedrichs $N$, Steiner $S$, Buettner $R$, Knoepfle G: Immunohistochemical expression patterns of AP2alpha and AP2gamma in the developing fetal human breast. Histopathology 2007, 51:814-823.

4. Gee JM, Robertson JF, Ellis 10, Nicholson RI, Hurst HC: Immunohistochemical analysis reveals a tumour suppressor-like role for the transcription factor AP-2 in invasive breast cancer. J Pathol 1999, 189:514-520.
5. Pellikainen J, Naukkarinen A, Ropponen K, Rummukainen J, Kataja V, Kellokoski J, Eskelinen M, Kosma VM: Expression of HER2 and its association with AP-2 in breast cancer. Eur J Cancer 2004, 40:1485-1495.

6. Bosher JM, Williams T, Hurst HC: The developmentally regulated transcription factor AP-2 is involved in c-erbB-2 overexpression in human mammary carcinoma. Proc Natl Acad Sci USA 1995, 92:744-747.

7. Turner BC, Zhang J, Gumbs AA, Maher MG, Kaplan L, Carter D, Glazer PM, Hurst HC, Haffty BG, Williams T: Expression of AP-2 transcription factors in human breast cancer correlates with the regulation of multiple growth factor signalling pathways. Cancer Res 1998, 58:5466-5472.

8. Pellikainen JM, Kosma VM: Activator protein-2 in carcinogenesis with a special reference to breast cancer-a mini review. Int J Cancer 2007, 120:2061-2067.

9. Bragança J, Eloranta JJ, Bamforth SD, Ibbitt JC, Hurst HC, Bhattacharya S: Physical and functional interactions among AP-2 transcription factors, p300/CREB-binding protein, and CITED2. J Biol Chem 2003, 278:16021-16029.

10. Bragança J, Swingler T, Marques FIR, Jones T, Eloranta JJ, Hurst HC, Shioda T, Bhattacharya S: Human CREB-binding protein/p300-interacting transactivator with ED-rich tail (CITED) 4, a new member of the CITED family, functions as a co-activator for transcription factor AP-2. J Biol Chem 2002, 277:8559-8565.

11. Bamforth SD, Braganca J, Eloranta JJ, Murdoch JN, Marques Fl, Kranc KR, Farza H, Henderson DJ, Hurst HC, Bhattacharya S: Cardiac malformations, adrenal agenesis, neural crest defects and exencephaly in mice lacking Cited2, a new Tfap2 co-activator. Nat Genet 2001, 29:469-474.

12. Jiang MS, Tang QQ, McLenithan J, Geiman D, Shillinglaw W, Henzel WJ, Lane MD: Derepression of the C/EBPalpha gene during adipogenesis: identification of AP-2alpha as a repressor. Proc Natl Acad Sci USA 1998, 95:3467-3471.

13. Wajapeyee N, Britto R, Ravishankar HM, Somasundaram K: Apoptosis induction by activator protein 2alpha involves transcriptional repression of Bcl-2. J Biol Chem 2006, 281:16207-16219.

14. Wang $X: A P-2$ : a regulator of EGF receptor signaling and proliferation in skin epidermis. J Cell Biol 2006, 172:409-421.

15. Eloranta JJ, Hurst HC: Transcription factor AP-2 interacts with the SUMOconjugating enzyme UBC9 and is sumolated in vivo. J Biol Chem 2002, 277:30798-30804.

16. Meier P, Koedood M, Philipp J, Fontana A, Mitchell PJ: Alternative mRNAs encode multiple isoforms of transcription factor AP-2 during murine embryogenesis. Dev Biol 1995, 169:1-14.

17. Limesand SW, Anthony RV: Novel activator protein-2alpha splice-variants function as transactivators of the ovine placental lactogen gene. Eur J Biochem 2001, 268:2390-2401.

18. Davuluri RV, Suzuki Y, Sugano S, Plass C, Huang TH: The functional consequences of alternative promoter use in mammalian genomes. Trends Genet 2008, 24:167-177.

19. Williams T, Admon A, Lüscher B, Tjian R: Cloning and expression of AP-2, a cell-type-specific transcription factor that activates inducible enhancer elements. Genes Dev 1988, 2:1557-1569.

20. Boyes J, Byfield P, Nakatani Y, Ogryzko V: Regulation of activity of the transcription factor GATA-1 by acetylation. Nature 1998, 396:594-598.

21. Soutoglou E, Katrakili N, Talianidis I: Acetylation regulates transcription factor activity at multiple levels. Mol Cell 2000, 5:745-751.

22. Hollywood DP, Hurst HC: A novel transcription factor, OB2-1, is required for overexpression of the proto-oncogene c-erbB-2 in mammary tumour lines. EMBO J 1993, 12:2369-2375.

23. Debnath J, Muthuswamy SK, Brugge JS: Morphogenesis and oncogenesis of MCF-10A mammary epithelial acini grown in three-dimensional basement membrane cultures. Methods 2003, 30:256-268.

24. ImageJ. [http://rsb.info.nih.gov/ij/]

25. lacono M, Mignone F, Pesole G: UAUG and uORFs in human and rodent 5'untranslated mRNAs. Gene 2005, 349:97-105.

26. Gong L, Kamitani T, Fujise K, Caskey LS, Yeh ET: Preferential interaction of sentrin with a ubiquitin-conjugating enzyme, Ubc9. J Biol Chem 1997, 272:28198-28201.

27. Vernimmen D, Begon D, Salvador C, Gofflot S, Grooteclaes M, Winkler R: Identification of HTF (HER2 transcription factor) as an AP-2 (activator protein-2) transcription factor and contribution of the HTF binding site to ERBB2 gene overexpression. Biochem J 2003, 370:323-329. 
28. Arpino G, Wiechmann L, Osborne CK, Schiff R: Crosstalk between the estrogen receptor and the HER tyrosine kinase receptor family: molecular mechanism and clinical implications for endocrine therapy resistance. Endocr Rev 2008, 29:217-233.

29. Geiss-Friedlander R, Melchior F: Concepts in sumoylation: a decade on Nat Rev Mol Cell Biol 2007, 8:947-956.

30. Ramos YF, Hestand MS, Verlaan M, Krabbendam E, Ariyurek Y, van Galen M, van Dam H, van Ommen GJ, den Dunnen JT, Zantema A, 't Hoen PA: Genome-wide assessment of differential roles for p300 and CBP in transcription regulation. Nucleic Acids Res 38:5396-5408.

doi: $10.1186 / \mathrm{bcr} 2838$

Cite this article as: Berlato et al: Alternative TFAP2A isoforms have distinct activities in breast cancer. Breast Cancer Research 2011 13:R23.

Submit your next manuscript to BioMed Central and take full advantage of:

- Convenient online submission

- Thorough peer review

- No space constraints or color figure charges

- Immediate publication on acceptance

- Inclusion in PubMed, CAS, Scopus and Google Scholar

- Research which is freely available for redistribution

Submit your manuscript at www.biomedcentral.com/submit 